\title{
Gold nanoparticles to improve HIV drug delivery
}

Background: Antiretroviral therapy (ART) has improved lifespan and quality of life of patients infected with the HIV-1. However, ART has several potential limitations, including the development of drug resistance and suboptimal penetration to selected anatomic compartments. Improving the delivery of antiretroviral molecules could overcome several of the limitations of current ART. Results \& Conclusion: Two to ten nanometer diameter inorganic gold crystals serve as a base scaffold to combine molecules with an array of properties in its surface. We show entry into different cell types, antiviral activity of an HIV integrase inhibitor conjugated in a gold nanoparticle and penetration into the brain in vivo without toxicity. Herein, gold nanoparticles prove to be a promising tool to use in HIV therapy.

The introduction of antiretroviral therapy (ART) into clinical practice in the 1990's led to a huge improvement in the life expectancy and quality of life of HIV-infected persons. During the first years of antiretroviral treatment, when only a few antiretroviral drugs were available, $<40 \%$ of treated patients achieved virologic suppression after a year of treatment [1]. Currently, 31 antiretroviral drugs are approved for the treatment of HIV infection, and virologic success rate is usually $>80 \%$, even when drug resistance is present [2].

However, a cure for HIV infection has not yet been described, and so lifelong antiretroviral treatment is needed by many, entailing risks of the emergence of drug resistance, long-term drug toxicities and loss of adherence to therapy over time. In addition, antiretroviral drugs fail to penetrate in certain tissues, allowing the creation of viral reservoirs. Thus, despite all the benefits that ART confers, improvements in ART can be made.

Nanomedicine is a promising area of biotechnology full of possibilities for novel therapeutics. Nanoparticles are primarily characterized by their size, in the nanometer range. This small size confers unique chemical and physical properties, useful in imaging, diagnosis and therapy. Several nanoparticle systems have already been approved for clinical use, primarily liposomal drugs and polymer-drug conjugates [3]. For HIV therapy, the existing HIV antiretroviral drugs indinavir, zidovudine and saquinavir have undergone nanoformulation for testing in vitro systems and preclinical animal models [4]. Antiretroviral drug combinations have also been nanoformulated, such as efavirenz, atazanavir and ritonavir [5], and efavirenz, lopinavir and ritonavir [6]. Both demonstrated robust antiviral effect and improved bioavailability.

Recently, we have become interested in the application of small molecule-conjugated inorganic nanoparticles, gold in particular, to generate potentially new therapeutics for the treatment of infectious diseases. In the present study, we tested gold nanoparticles (AuNPs) for the treatment of HIV.

Gold nanoparticles have already been used in gene and cancer targeting, imaging and delivery of therapeutics [7-10], reaching clinical trials for cancer patients [11]. Several characteristics make AuNPs highly attractive for clinical use, such as their small size that facilitates entry into tissues and cells, their inert nature that insures little host response to the molecules, and their potential for
Carolina Garrido1, Carrie A Simpson², Noelle P Dahl', Jamee Bresee ${ }^{2}$, Daniel C Whitehead $^{3}$, Erick A Lindsey ${ }^{3}$, Tyler L Harris ${ }^{3}$, Candice A Smith², Carly J Carter², Daniel L Feldheim², Christian Melander ${ }^{3}$ \& David M Margolis*,1,4,5

'Department of Medicine, University of North Carolina at Chapel Hill, Chapel Hill, NC 27599-7005, USA

${ }^{2}$ Department of Chemistry, University of Colorado, Boulder, CO 80309, USA

${ }^{3}$ Department of Chemistry, North Carolina State University, Raleigh, NC 27607, USA

${ }^{4}$ Department of Microbiology \& Immunology, University of North Carolina at Chapel Hill, Chapel Hill, NC 27599, USA

${ }^{5}$ Department of Epidemiology, University of North Carolina at Chapel Hill, Chapel Hill, NC 27516, USA

*Author for correspondence: Tel.: +1 9199666388 dmargo@med.unc.edu

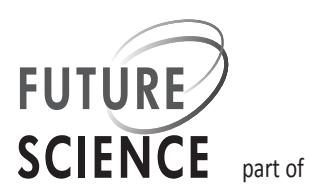




\section{Key terms}

Antiretroviral therapy: Combination of three or more drugs used to treat HIV infection and suppress viral replication. Drugs target different steps in the viral cycle to inhibit them, such as viral entry, retrotranscription, integration and maduration.

HIV: Causal agent of the AIDS.

Viral reservoir: Cell or anatomical site where a replicationcompetent viral genome persists in a latent state from which can be reactivated and produce virions.

Blood-brain barrier: Selective permeability barrier which separates the central nervous system from the circulating blood. It is comprised by capillary endothelial cells connected by tight junctions.

Raltegravir: First in class of the family of antiretrovirals inhibiting the integrase of the HIV. Specifically inhibits the strand transfer step within integration of the viral genome in the host chromosome. Marketed by Merck and first approved by the US FDA in 2007.

multivalency which allows the simultaneous conjugation of different molecules in the nanoparticle surface and the simultaneous delivery of these payloads. Herein, we study the capacity of AuNPs to enter into different cell types, cross the blood-brain barrier (BBB) and exert antiviral activity upon conjugation with an antiretroviral.

\section{Methods}

\section{Preparation of AuNPs}

P-mercaptobenzoic acid (pMBA) coated AuNPs were synthesized according to our previous publications [12,13]. A solution of $20 \mathrm{mM} \mathrm{HAuCl}$ (Strem, MA, USA) dissolved in $20 \mathrm{ml}$ of methanol was combined with $85.0 \mathrm{mM}$ pMBA dissolved in $\mathrm{pH} 12$ ultrapure water. Gold mixtures were allowed to equilibrate for $15 \mathrm{~min}$ while stirring. The solutions $(0.40 \mathrm{mmol}$ of $\mathrm{Au}^{3+}$ ) were diluted to a final $\mathrm{Au}^{3+}$ concentration of $0.55 \mathrm{mM}$ with the addition of $202 \mathrm{ml}$ of ultrapure water and $186 \mathrm{ml}$ of methanol. The $\mathrm{Au}^{3+}$ was reduced with $7.2 \mathrm{ml}$ of a $0.25 \mathrm{M}$ aqueous sodium borohydride (Sigma-Aldrich, MO, USA) solution. The reduction was allowed to proceed for $24 \mathrm{~h}$ at room temperature with constant stirring. Gold nanoparticles were precipitated with the addition of $120 \mathrm{mmol}$ of $\mathrm{NaCl}$ in $720 \mathrm{ml}$ of methanol followed by centrifugation at $3200 \times g$ RCF for 5 min. Precipitated nanoparticles were reconstituted in water.

The concentration was measured by UV-visible spectroscopy using the extinction coefficient of $400,000 \mathrm{M}^{-1 \mathrm{~cm}-1}$ at $510 \mathrm{~nm}$.

\section{Place-exchange of ligands to AuNPs}

One pot place exchange reactions were conducted with the addition of varying concentrations of ligand of interest - raltegravir, Cy5, TAMRA or glucose - to a $10 \mu \mathrm{M}$ concentration of AuNPs in $20 \mathrm{mM}$ sodium phosphate buffer, $\mathrm{pH}$ 9.5. Reactions were placed on a plate shaker and agitated for $24 \mathrm{~h}$ at room temperature. The exchange product was harvested through the addition of 40 mmoles of $\mathrm{NaCl}$ and a volume of methanol equal to that of phosphate buffer and added salt. Reactions were centrifuged at 3200 $\times g$ RCF for 30-60 min. Precipitated nanoparticles were resuspended and precipitated with the addition of $\mathrm{NaCl}$ and methanol 2-times to wash out excess unreacted thiol. Particles were allowed to dry to completion overnight at room temperature and resuspended in $20 \mathrm{mM}$ sodium phosphate buffer, $\mathrm{pH}$ 9.5. Resuspended nanoparticles were washed with $20 \mathrm{mM}$ sodium phosphate buffer, $\mathrm{pH} 9.5$ over a 30K MWCO centricon filter to remove excess salt and thiol. The final concentrations were determined by UV-visible spectroscopy as described above.

\section{Characterization of AuNPs}

\section{Transmission electron microscopy}

Transmission electron microscopy (TEM) grids were prepared by placing a drop of the AuNPs on a carbon film covered copper mesh grid for a minute, and then excess solution was wicked away with a paper filter. Grids were then allowed to air-dry for $45 \mathrm{~min}$ before being imaged by TEM. The subsequent TEM images were analyzed by Image-J (National Institutes of Health, MA, USA) to determine the size distribution of the AuNP cores. A minimum of 100 particles was measured before analysis of size distribution.

\section{Analysis of place-exchange ratios}

The success of place-exchange was measured using our previous methods [13] for UV-vis with no exceptions. Measurements were recorded at wavelengths of 260 and $510 \mathrm{~nm}$.

\section{In vitro cellular uptake \& toxicity of AuNPs Immunohistochemistry}

Uptake of AuNPs was evaluated in peripheral blood mononuclear cells (PBMCs), macrophages and human brain microendothelial cells (HBMECs). PBMCs were obtained by Ficoll gradient centrifugation, and macrophages were selected by adherence to plastic. HBMECs were primary cell lines obtained by elutriation of dispase-dissociated normal human brain cortex tissue (Cell-Systems, WA, USA).

PBMCs and macrophages were incubated for $24 \mathrm{~h}$ with $500 \mathrm{nM}$ AuNPs conjugated with Cy5 or TAMRA in Iscove's Modified Dulbecco's Media (IMDM) supplemented with penicillin/streptomycin, $10 \%$ fetal bovine serum (FBS) and $5 \mathrm{U} / \mathrm{ml}$ recombinant IL-2 (Peprotech, NJ, USA). HBMECs were incubated for 
24 h with $100 \mathrm{nM}$ AuNPs conjugated with TAMRA in CSC Complete Medium (Cell-Systems, WA, USA) with $10 \%$ FBS. Cells were then washed 3-times with phosphate buffered saline (PBS) and stained to allow nuclei visualization with Hoechst 33342 (ThermoScientific, IL, USA) or DAPI (Molecular Probes, Invitrogen, CA, USA). In addition, cells were stained with CellMask Orange plasma membrane stain (Molecular Probes, Invitrogen, Carlsbad, CA) to allow membrane visualization. HBMECs were stained with anti-B-catenin (Sigma-Aldrich) instead of CellMask to visualize tight junctions. After washing with PBS, cells were plated in chambered cover glasses (Lab-Tek, Thermo Fisher Scientific, Inc., Roskilde, Denmark) and imaged by confocal laser scanning microscopy (Olympus FV1000 Multiphoton Confocal, Olympus America, PA, USA), using the $60 \times$ objective.

\section{Flow cytometry}

PBMCs were incubated with IMDM supplemented with penicillin/streptomycin, 10\% FBS and $5 \mathrm{U} / \mathrm{ml}$ IL-2 during 2 days. Gold nanoparticles conjugated with Cy5 at different concentrations $(0,1$ and $3 \mu \mathrm{M})$ were added to the plates and harvested after 6 and $24 \mathrm{~h}$. Samples were washed twice with PBS 2\% FBS and then stained with Live/Dead Fixable Aqua Dead Cell Stain (Molecular Probes, Invitrogen, NY, USA) following manufacturer's instructions. Flow cytometry was performed using BD LSR Fortessa equipped with FACSDIVA software. PBMCs were gated by forward scatter versus sideward scatter (FSC/SSC) plots, and gated on the viable population according to Aqua Dead Cell staining, selecting the negative fraction. Nanoparticle uptake was quantified on this viable population by $\mathrm{Cy} 5$ fluorescence.

To further analyze cell toxicity, we incubated CD8depleted PBMCs with increasing concentrations of AuNPs conjugated with raltegravir or AuNPs alone for 24, 48 and $72 \mathrm{~h}$. We selected CD8-depleted cells because HIV inhibition assays are performed in this cell population, given that CD8 cells naturally secrete antiviral factors and exert considerable antiviral activity. Thus, it was important to assess safety particularly in CD8-depleted cells. After the different incubation times, cells were harvested and stained with FITCAnnexin V and 7AAD (Biolegend, CA, USA) and analyzed by fluorescence-activated cell sorter (FACS). We considered cells positive for both Annexin V and 7-AAD as the dead population, without differentiating degrees of apoptosis/necrosis.

\section{In vivo delivery of AuNPs to the brain}

Female adult BALB mice were used to analyze the distribution of the AuNPs in vivo. All animal work was approved by the University of North Carolina Institutional Animal Care and Use Committee.

Gold nanoparticles were diluted in $200 \mu \mathrm{l}$ PBS at a concentration of $30 \mu \mathrm{M}$ to be injected into the tail vein of each of three mice. In addition, a mouse was used as negative control and was injected with a PBS solution without nanoparticles. After $24 \mathrm{~h}$, animals were humanely euthanized, and tissues were harvested. Organs were collected in PBS, weighed and digested with nitric acid (Optima, Fisher Scientific, Roskilde, Denmark) overnight. Samples were heated until all acid had evaporated, and tissues were resuspended in water, sonicated to eliminate any remaining tissue and analyzed using inductively coupled plasma - optical emission spectroscopy [14] to quantitate the gold content. The same procedure was performed using AuNPs conjugated with glucose.

\section{ICP-MS analysis}

Analysis of gold content in biological samples was performed using a Perkin-Elmer SCIEX ICP-MS (model Elan DRC-e, IL, USA) at the University of Colorado Laboratory for Environmental and Geological Sciences. Statistical analysis of samples was performed as described in Simpson et al. [15] involving the student's $t$ test (95\% confidence level), but with units in $\mu \mathrm{g} / \mathrm{ml}$. The detection limit of the instrument was $0.02 \mathrm{ppb}$ (0.02 ng Au/ml) sample.

\section{HIV inhibition with AuNPs conjugated with raltegravir}

Raltegravir (RAL) was modified with the incorporation of a thiol group to serve as linker to a gold nanoparticle, designated EL-04-109 or thiolated raltegravir (Figure 1). Gold nanoparticles were conjugated to different number of molecules of this RAL derivative, making particles with 4 (4:1), 33 (33:1) and maximal numbers (60:1) of EL-04-109 RAL derivative molecules. CD8depleted PBMCs from a healthy donor were stimulated with $2 \mu \mathrm{g} / \mathrm{ml}$ phytohematoglutinin (Remel, KS, USA) and $60 \mathrm{U} / \mathrm{ml}$ of IL-2 for $24 \mathrm{~h}$. Drugs were diluted to different concentrations and added to the cells in triplicate. Finally, cells were infected with the R5 tropic HIV strain JRCSF (16 pg p24/10 cells). Supernatants were harvested at day 5 and HIV-1 capsid p24 antigen was measured by ELISA (ABL, MD, USA).

\section{Raltegravir derivatives synthesis}

Detailed explanation of raltegravir derivatives synthesis can be found in the Supporting Material section.

\section{Results}

\section{Characterization of the AuNPs}

The diameter of the pMBA-Au nanoparticles was $1.8 \pm 0.32 \mathrm{~nm}$ as determined by TEM. This size is 


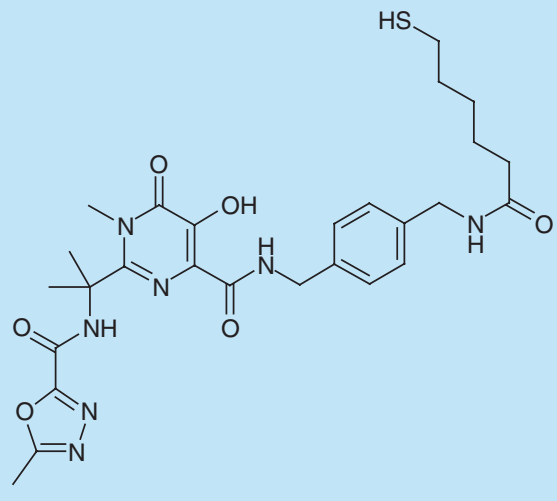

Figure 1. Modified raltegravir molecule, EL-04-109 (thiolated raltegravir). Chemical formula: $\mathrm{C}_{27} \mathrm{H}_{35} \mathrm{~N}_{7} \mathrm{O}_{6} \mathrm{~S}$. Exact mass: 585.2370. Molecular weight: 585.6751.

similar to pMBA-Au nanoparticles characterized by $\mathrm{X}$-ray crystallography to have a molecular formula of $\mathrm{Au}_{144}(\mathrm{pMBA})_{60}$ (Figure 2).

For the HIV inhibition assays, AuNPs were conjugated with different numbers of thiolated raltegravir molecules, resulting in particles with 4,33 and complete coverage (60).

\section{Cellular uptake \& toxicity of AuNPs}

Cellular uptake of fluorescence-labeled nanoparticles was qualitatively evaluated by confocal laser scanning microscopy. Gold nanoparticles were conjugated with $\mathrm{Cy} 5$ or TAMRA (AuNP-Cy5 or AuNP-TAMRA, respectively) and incubated with different cell types - PBMCs, macrophages and HBMECs - to evaluate cellular uptake. Confocal microscopy imaging showed nanoparticles

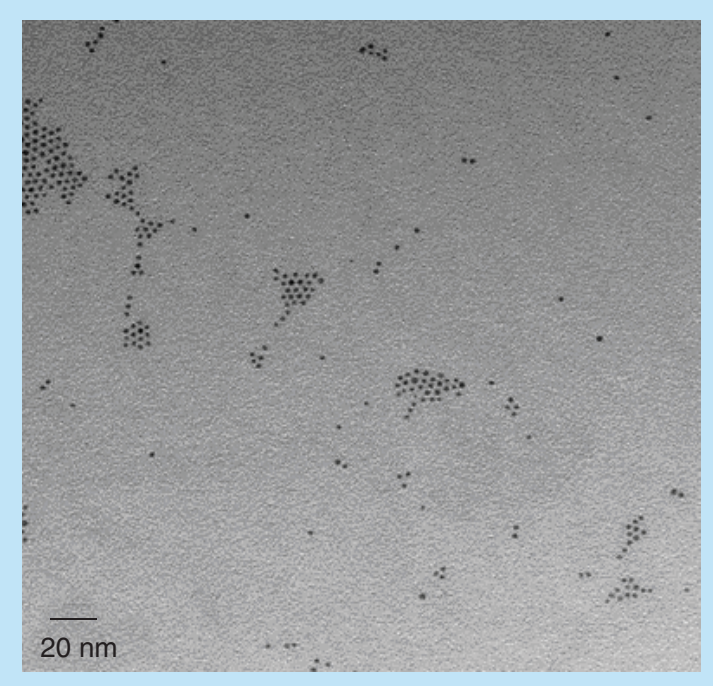

Figure 2. Transmission electron microscopy image of gold-p-mercaptobenzoic acid nanoparticles. Particles displayed an average diameter of $1.8 \pm 0.32 \mathrm{~nm}$. inside all the three cell types after $24 \mathrm{~h}$ (Figure 3). Fluorescence was observed widely in the cytoplasm of all the cells, but signal in the nucleus was much weaker.

To determine more accurately the uptake kinetics and to evaluate cell toxicity, PBMCs were incubated with increasing concentrations of AuNP-Cy5 and analyzed with FACS at different time points. FACS data indicated AuNPs cellular uptake in a dose-time dependent way (Figure 4). We observed a gradual increase in the intensity of $\mathrm{Cy} 5$ in the viable cell population, reaching $54 \%$ after $24 \mathrm{~h}$ incubation with $3 \mu \mathrm{M}$ nanoparticles. This indicates a progressive cellular uptake of the nanoparticles. In addition, after incubating PBMCs with different concentrations of AuNPs conjugated or not with raltegravir, a complete lack of toxicity was observed at 24, 48 and $72 \mathrm{~h}$ (Figure 5).

\section{In vivo delivery to the brain}

Three mice were injected via tail vein with nanoparticles at a concentration of $30 \mu \mathrm{M}$. After $24 \mathrm{~h}$, mice were sacrificed and tissues were extracted for gold quantification by inductively coupled plasma-mass spectrometry (ICP-MS). ICP-MS gold measurements revealed that the majority of nanoparticles accumulate in the spleen, reaching up to $28 \%$ of the injected material per gram of tissue in one of the mice. Au was also found in liver, kidneys, lung, tail, heart, brain and blood (Table 1). To increase nanoparticle transport across the $\mathrm{BBB}$, we conjugated glucose to the AuNPs, given that BBB express Glut-1 receptor, which mediates glucose transport. Average brain content of Au was $333.6 \mathrm{ng} / \mathrm{g}$ of tissue, and it did not differ between mice injected with AuNP-TAMRA and AuNP-glucose (347 vs $320 \mathrm{ng} / \mathrm{g}$ tissue, respectively). However, upon conjugation of glucose to the nanoparticles, accumulation into the spleen seemed to decrease (34,356 vs $15,074 \mathrm{ng} / \mathrm{g}$ tissue in the AuNP and AuNP-glucose, respectively).

\section{HIV inhibition with AuNPs conjugated with raltegravir}

Gold nanoparticles were conjugated with molecules of raltegravir and HIV inhibition was tested in infected primary PBMCs depleted from CD8+cells. As shown in Figure $6 \mathrm{~A}$, at day 5 , AuNPs conjugated with four molecules of raltegravir (RAL-AuNP 4:1) inhibited HIV-1 replication down to $25.23 \%$. Interestingly, increasing the number of RAL molecules into each gold particle did not improve antiviral performance but rather impaired it, as shown when tested 33:1 or 60:1 (RAL:AuNP). As with our previous work [13], it is important to note the lack of antiviral activity of free modified raltegravir, due to the alteration of its structure, and that activity was restored when the molecule was conjugated to the gold particle. Commercial raltegravir, used as positive control, 


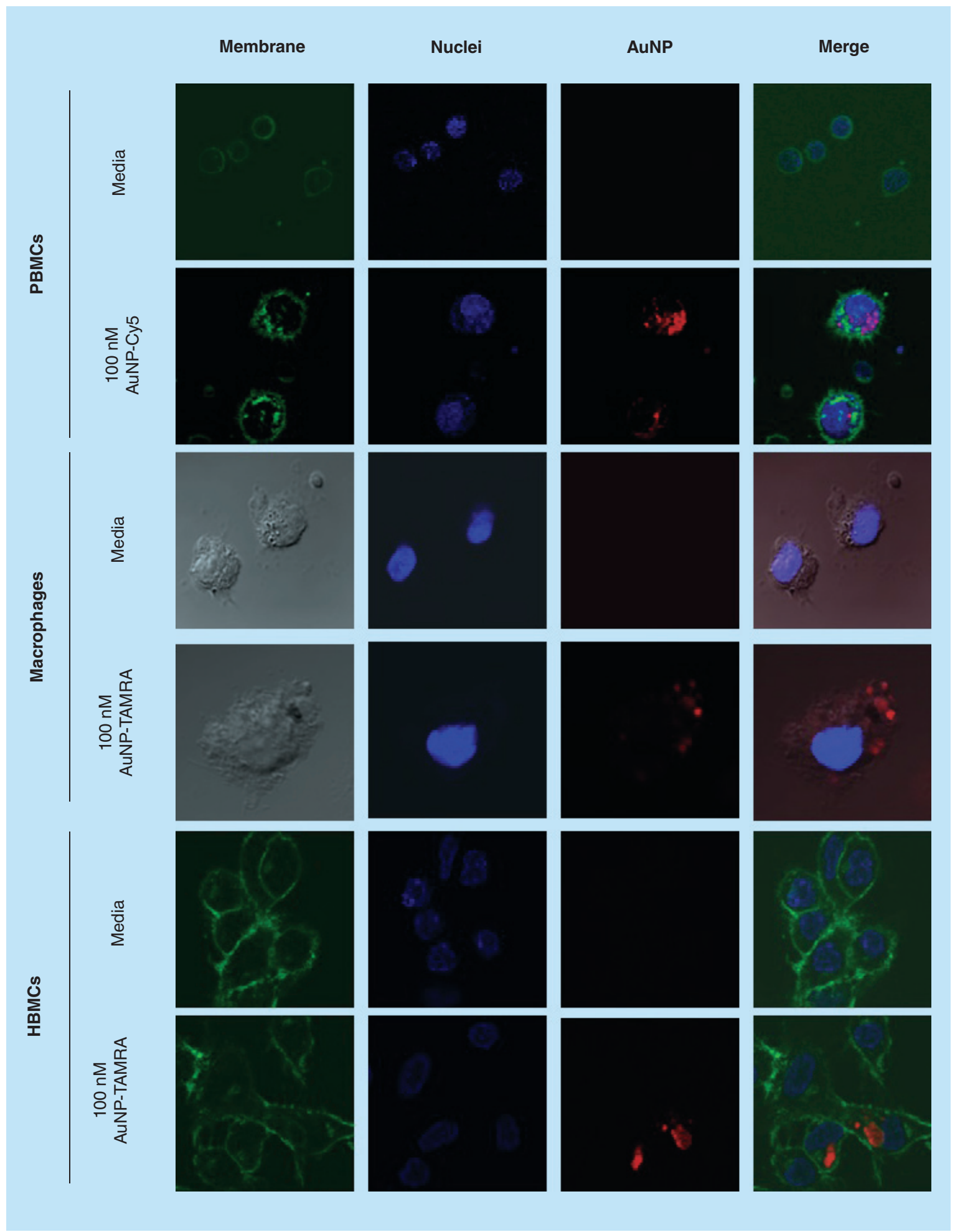

Figure 3. Confocal images of different cell types incubated with gold nanoparticles-tetramethylrhodamine or gold nanoparticles-Cy5 (red) for $24 \mathrm{~h}$ at $37^{\circ} \mathrm{C}$ on glass coverslips and fixed before mounting. Nuclei were stained with 4',6-diamidino-2-phenylindole or Hoechst 33342 (blue). Transmitted light overlay, Cell Mask stain or $\beta$-catenin (green) shows the delimited plasma membrane for each cell type. Images are from a representative view of the whole picture and from one of the at least three experiments performed.

AuNP: Gold nanoparticle; HBMC: Human brain microendothelial cell; PBMC: Peripheral blood mononuclear cell; TAMRA: Tetramethylrhodamine. 


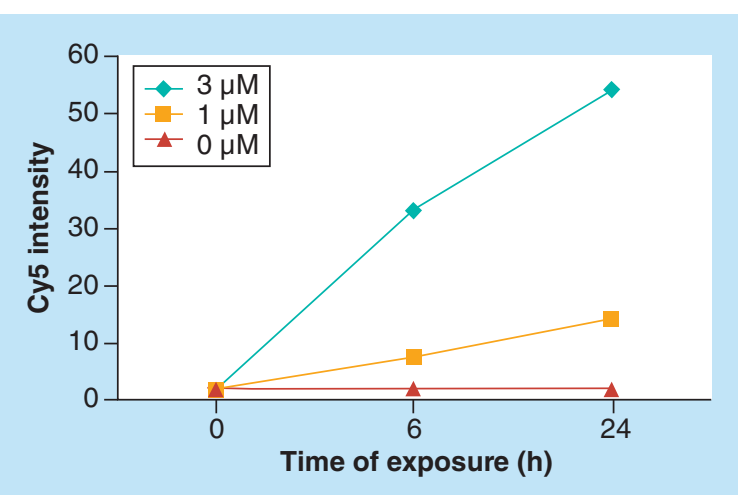

Figure 4. Cy5 intensity measured by FACS after peripheral blood mononuclear cells were exposed for 6 or $\mathbf{2 4} \mathrm{h}$ to gold nanoparticles-Cy5. Peripheral blood mononuclear cells were derived from one healthy donor. Cell-associated fluorescence was evaluated using FACS analysis, gating on the viable population. A steady increase over time in the associated fluorescence was observed and it was also concentration dependent. FACS: Fluorescence-activated cell sorter.

inhibited viral replication at lower concentrations. We also tested the impact of AuNPs without RAL conjugation on HIV replication in an independent experiment. As shown in Figure 6B, AuNPs themselves did not have a significant effect on HIV replication, and thus we confirm that the effect observed with AuNP-RAL molecules is caused by the addition of RAL to the gold core.

\section{Discussion}

Nanotechnology is a novel and promising tool for diagnosis and treatment in medicine. Different materials such as dendrimers, liposomes, synthetic polymeric nanoparticles, micelles or inorganic substances (iron oxide, quantum dots, gold and silica) can be fabricated for use in nanoscale.

Here, we have studied the properties of AuNPs to assess their potential use for HIV therapy. We have proven that AuNPs can be conjugated to different molecules and penetrate into cells. They can also cross the BBB allowing the transport of molecules to the brain. Moreover, cell viability assays showed that these AuNPs did not cause short-term cell toxicity and that they can be conjugated to an antiviral molecule and retain antiviral activity. We have previously demonstrated the latter with an antiviral nanoparticle that acted outside of the cell, blocking viral entry [13], but here we demonstrate the activity of an antiviral nanoparticle that acts inside the cell, which is of importance given that many drugs need to be inside the cell to exert their activity. Such capabilities have been individually shown for nanoparticles $[13,15,16]$, but this is the first demonstration of a multifunctional AuNP. These results support continued studies of AuNPs as scaffolds for the delivery of therapeutic molecules.

In the present work, we first tested the ability of AuNPs to enter different cell types. Cellular uptake efficiency depends on both characteristics of the nanoparticle, such as size, shape and charge of the surface, and properties of the cell, such as the cell cycle state [17]. Additionally, cellular uptake and partitioning into cellular organelles and domains (e.g., nuclear vs cytoplasmic) can each differ between cell types [18]. We investigated nanoparticle entry into different cells types, but focused on entry into lymphocytes, as this is the principal cell type involved in

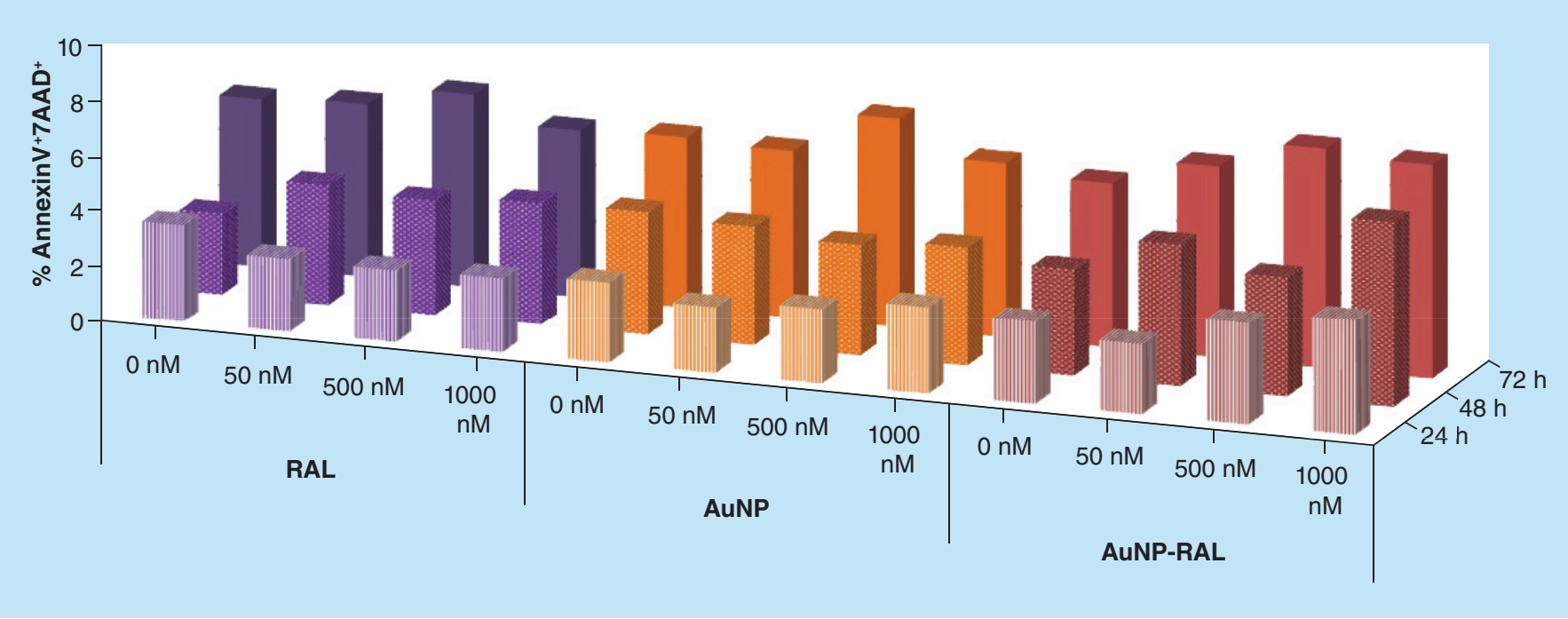

Figure 5. Cell viability measured by FACS after 24,48 and $72 \mathrm{~h}$ incubation with raltegravir, gold nanoparticle or gold nanoparticle-raltegravir at different concentrations $(\mathbf{0}, \mathbf{5 0}, \mathbf{5 0 0}$ and $\mathbf{1 0 0 0} \mathrm{nM})$. Peripheral blood mononuclear cells were derived from one healthy donor. Graph represents percentage of peripheral blood mononuclear cells positive for both annexin $V$ and 7-aminoactinomycin D, and as shown, viability is not compromised by culture with the different nanoparticles in this 3-day period. AuNP: Gold nanoparticle; FACS: Fluorescence-activated cell sorter; RAL: Raltegravir. 
Table 1. Gold content in different mouse tissues $24 \mathrm{~h}$ after $30 \mu \mathrm{M}$ gold nanoparticle tail injection (three different mice per condition).

\begin{tabular}{lll|}
\hline Tissue & AuNP, $\mu$ g/g (SD) & AuNP-glucose, $\mu$ g/g (SD) \\
\hline Spleen & $34.356(1.112)$ & $15.074(2.548)$ \\
\hline Liver & $6.991(1.810)$ & $5.353(2.476)$ \\
\hline Kidney & $5.934(1.671)$ & $4.910(1.744)$ \\
\hline Tail & $4.508(2.077)$ & $2.830(0.738)$ \\
\hline Lungs & $1.985(0.260)$ & $1.967(0.191)$ \\
Heart & $3.028(0.022)$ & $1.513(0.284)$ \\
\hline Brain & $0.347(0.050)$ & $0.320(0.131)$ \\
\hline Muscle & $0.867(0.400)$ & $0.083(0.083)$ \\
\hline Blood & $1.993(0.167)$ & $3.062(0.345)$ \\
\hline $\begin{array}{l}\text { Gold content was measured by inductively coupled plasma-MS. AuNPs tend to accumulate in spleen and liver, probably due to } \\
\text { reticuloendothelial system clearance. Glucose conjugation did not change the distribution profile. } \\
\text { AuNP: Gold nanoparticle. }\end{array}$ \\
\hline
\end{tabular}

HIV infection. Using fluorophore-conjugated nanoparticles, we observed by confocal microscopy that nanoparticles could enter not only into lymphocytes but also into other cell types of potential importance in HIV disease, such as macrophages and brain microendothelial cells. Mechanism of entry into the different cells types might differ [19]. Entry into macrophages would be expected to occur at least in part via phagocytosis, while entry into lymphocytes may occur via direct entry or pinocytosis. Particles were widely observed in the cytoplasm, but partition into the nucleus was often reduced. Although for many applications cytoplasmic delivery is sufficient, nuclear entry will be required for others. Nanoparticle size is important for entry into the nucleus, as the size of the nuclear pore does not exceed $45 \mathrm{~nm}$ [20]. Gold nanoparticles such as those used in this study can be fabricated at diameters of approximately $2 \mathrm{~nm}$. However, if facilitated entry into the nucleus is required, this might be achieved by attaching nuclear entry or targeting signals such as peptides from the SV40 large T antigen [21] or the HIV Tat peptide [22].

In theory, the in vivo administration of AuNPs might elicit toxicity and immunogenicity [23]. Thus, it is essential to evaluate their safety. Previous studies have demonstrated AuNPs are well tolerated in vivo [15], and in vitro toxicity may be primarily related to moieties attached to the nanoparticle, rather than the gold particle itself [12,13,18,24-26]. Here, when administered to mice, no symptoms of toxicity were seen over $24 \mathrm{~h}$. In vitro toxicity was preliminarily evaluated by measuring a marker of early apoptosis over 3 days of exposure, and we found no evidence of toxicity.

The use of nanoparticle drug delivery systems to improve the efficiency of delivery of antiretroviral drugs such as saquinavir [27,28], atazanavir [29] and even a combination of different antiretroviral drugs [5,6] have been reported. However, these systems simply encapsulated the unmodified drugs inside particles, for improved cellular delivery.

Very few approaches using AuNPs as carrier for anti-HIV drugs have been reported [30,31]. Our groups tested in a previous work that AuNPs conjugated to a CCR5 antagonist, reduced viral production [13]. However, given the mechanism of action of CCR5 antagonists, that is, viral entry inhibition, it was not demonstrated that nanoparticles could enter into the cell and exert antiviral activity. In the present study, we conjugated AuNPs with a modified form of raltegravir that was only active when conjugated to gold. By thiolating the compound, we introduce a steric hindrance that cripples the compound as a single molecular entity. However, once placed on a particle, the multivalent effect overcomes this penalty and delivers a viable entity. Thus, we could presume that a multivalent interaction is necessary that is not possible with the monomeric thiolated raltegravir but is present with the nanoparticles. The ability of these particles to inhibit HIV replication implies that these AuNPs enter the cell to exert antiviral effect. Interestingly, attaching a larger number of RAL molecules to the gold core, did not increase viral inhibition, as particles displaying 33 RAL molecules or more actually possessed less antiviral activity than nanoparticles carrying only $4 \mathrm{RAL}$ molecules. Many possible explanations for this attenuation of effect require further study, but it is possible that as the coverage of raltegravir on the nanoparticles increases, molecular gathering hinders interaction with the target by unavoidable van der Waals interactions.

HIV penetrates into the CNS and can cause neurocognitive disorders, even in the presence of antiretroviral treatment [32]. In addition, it has been shown that the CNS can act as an HIV reservoir [33], 


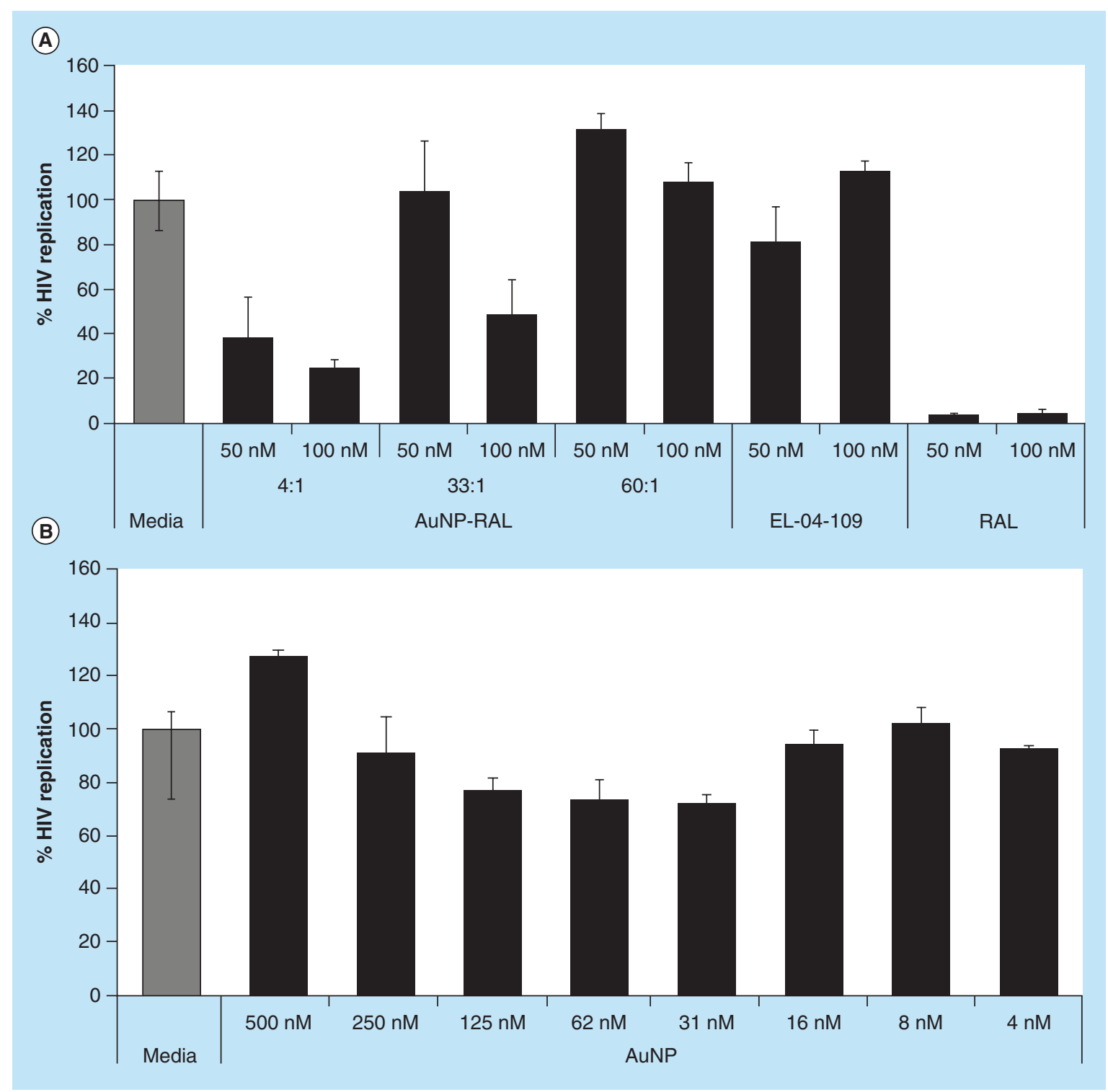

Figure 6. Percentage of HIV replication measured by HIV p-24 ELISA at day 5 of culture in HIV-infected CD8-depleted peripheral blood mononuclear cells derived from one healthy donor. $100 \%$ corresponds to the viral replication observed in the absence of any drug (mean \pm standard error of the mean). (A) AuNPs were conjugated with RAL at different ratios RAL:Au particle (4:1, 33:1, 60:1). After 5 days of culture with infected CD4+T cells, AuNP-RAL were able to inhibit viral replication to $25 \%$. (B) AuNPs without raltegravir conjugation were tested to analyze their antiviral activity at different concentrations (500-4 nM). After 5 days of culture with infected CD4 ${ }^{+}$ $T$ cells, no significant viral inhibition was observed in the presence of AuNPs.

AuNP: Gold nanoparticle; RAL: Raltegravir.

and thus, an important aim of our project was to improve drug delivery into the brain through the use of AuNPs. In this, we met only limited success. Previous studies have shown a relationship between particle size and biodistribution in murine models [34,35]. Intravenous administration of AuNPs of different sizes revealed a differential distribution among tissues, showing that smaller nanoparticles (i.e., $15 \mathrm{~nm}$ ) were more broadly distributed than larger ones (i.e., $200 \mathrm{~nm}$ ). As the nanoparticles increased their size, an increase in spleen accumulation and decrease in brain concen- tration was particularly observed. Thus, particle size has shown to be a factor in brain penetration, and we expected nanoparticles below $10 \mathrm{~nm}$ to enter the CNS more efficiently. However, in the mouse experiments we performed, AuNPs were predominantly found in spleen and only present in the brain at modest concentrations. Nanoparticles were capped with mercaptobenzoic acid, which enlarged their size somewhat, and may have impaired CNS penetration to some extent. The substantial distribution of AuNPs into the spleen was not surprising given the phagocytic function of 
this reticuloendothelial system (RES) organ, whose function is to remove foreign substances from the body. Some modifications can be made to the surface of the nanoparticles to protect them from RES clearance. The most widely used strategy is to coat the surface of the particle with surfactants such as polysorbate- 80 or albumin [36,37]. In order to reduce RES sequestration, we conjugated polyethyleneglycol to particles, to prolong their circulation time [38]. However, adding polyethyleneglycol of different lengths (data not shown) did not increase brain penetration or decrease spleen uptake. The transport of AuNPs across the BBB might occur through different mechanisms: transcellular lipophilic diffusion, transporter mediated transcytosis, paracellular hydrophilic diffusion, receptor mediated endocytosis or absorptive mediated endocytosis [39]. If transport takes place through receptor or transporter mediated endocytosis, we hypothesized that attaching a molecule that naturally crosses the BBB through specific receptors would help internalizing the whole nanoparticle complex. We conjugated glucose to the nanoparticles, but no improvement was observed. Finally, we conjugated a targeting peptide to try to direct nanoparticle delivery to the brain, but again we did not observe enhanced brain penetration. However, even if we did not manage to improve CNS penetration through any of the interventions, AuNPs were consistently found in the brain, proving that they are able to cross the BBB. More invasive approaches have been proposed to increase drug permeability through the $\mathrm{BBB}$, such as inducing changes in the permeability of the BBB by modifying the osmotic pressure [40] or disrupting the BBB with ultrasound [41]. However, such approaches carry with them clinical risks that are not acceptable, at least in the area of antiretroviral therapy.

\section{Conclusion}

In summary, we have demonstrated delivery of a small molecule-conjugated AuNP into the cell types in which HIV might replicate, and shown antiviral activity in HIV infected primary lymphocytes. We have also demonstrated delivery of gold particles into the CNS in an in vivo model, although entry is suboptimal. This leaves important future possibilities for the translational development of this technology, as the delivery of multiple therapeutic moieties on a single nanoparticle is technically feasible. Future advances could include antiviral particles that carry CNS-targeting molecules, or other molecules with novel or useful function.

\section{Future perspective}

The present study proposes the use of AuNPs to deliver antiretroviral drugs for HIV treatment. Here we demonstrate the ability of AuNPs to be conjugated to different molecules, such as raltegravir, and exert antiviral effect inside the cell. Next steps will include conjugating several different molecules to the AuNPs, allowing for simultaneous delivery of different therapeutics in the site of action, as well as evaluating the cellular uptake of AuNPs conjugated with different number and type of molecules.

\section{Supplementary data}

To view the supplementary data that accompany this paper please visit the journal website at: www.future-science.com/ doi/full/10.4155/FMC.15.57

\section{Acknowledgements}

We thank T Nochi and the the Michael Hooker Microscopy Facility personnel, M Chua and N Kramarcy for confocal microscopy support.

\section{Financial \& competing interests disclosure}

This work was supported by NIH grant R01 MH085597. The authors have no other relevant affiliations or financial involvement with any organization or entity with a financial interest in or financial conflict with the subject matter or materials discussed in the manuscript apart from those disclosed.

No writing assistance was utilized in the production of this manuscript.

\section{Ethical conduct of research}

The authors state that they have obtained appropriate institutional review board approval or have followed the principles outlined in the Declaration of Helsinki for all human or animal experimental investigations. In addition, for investigations involving human subjects, informed consent has been obtained from the participants involved.

\section{Executive summary}

- Synthesis of gold nanoparticles (AuNPs) with a size of 2-10 nm which can be conjugated to different molecules.

- AuNPs conjugated to fluorophores enter into peripheral blood mononuclear cells, macrophages and human brain microendothelial cells.

- AuNPs can reach the brain after vein tail injection in mice.

- AuNPs can be conjugated to an antiretroviral drug, raltegravir, and exert antiviral activity inside the cells. 


\section{References}

Papers of special note have been highlighted as:

- of interest; $\bullet \bullet$ of considerable interest

1 Lucas GM, Chaisson RE, Moore RD. Highly active antiretroviral therapy in a large urban clinic: risk factors for virologic failure and adverse drug reactions. Ann. Intern. Med. 131(2), 81-87 (1999).

2 Yazdanpanah Y, Fagard C, Descamps D et al. High rate of virologic suppression with raltegravir plus etravirine and darunavir/ritonavir among treatment-experienced patients infected with multidrug-resistant HIV: results of the ANRS 139 TRIO trial. Clin. Inf. Dis. 49(9), 1441-1449 (2009).

3 Zhang L, Gu FX, Chan JM, Wang AZ, Langer RS, Farokhzad OC. Nanoparticles in medicine: therapeutic applications and developments. Clin. Pharmacol. Ther. 83(5), 761-769 (2007).

- Comprehensive review of the state of the art in nanoparticle use for therapeutics.

4 Mahajan SD, Aalinkeel R, Law WC et al. Anti-HIV-1 nanotherapeutics: promises and challenges for the future. Int J. Nanomedicine 7, 5301-5314 (2012).

5 Nowacek A, Mcmillan J, Miller R, Anderson A, Rabinow B, Gendelman H. Nanoformulated antiretroviral drug combinations extend drug release and antiretroviral responses in HIV-1-infected macrophages: implications for neuroaids therapeutics. J. Neuroimmune Pharmacol. 5(4), 592-601 (2010).

- Interesting approach of antiretroviral nanoformulations using a cell-based delivery system to improve distribution.

6 Shibata A, Pham A, Belshan M et al. Polymeric nanoparticles containing combination antiretroviral drugs for HIV type 1 treatment. AIDS Res. Hum. Retroviruses 29(5), 746-754 (2013).

7 Rosi NL, Giljohann DA, Thaxton CS, Lytton-Jean AKR, Han MS, Mirkin CA. Oligonucleotide-modified gold nanoparticles for intracellular gene regulation. Science 312(5776), 1027-1030 (2006).

8 Thaxton CS, Georganopoulou DG, Mirkin CA. Gold nanoparticle probes for the detection of nucleic acid targets. Clin. Chim. Acta 363(1-2), 120-126 (2006).

9 Loo C, Hirsch L, Lee M-H et al. Gold nanoshell bioconjugates for molecular imaging in living cells. Opt. Lett. 30(9), 1012-1014 (2005).

10 Gibson JD, Khanal BP, Zubarev ER. Paclitaxel-functionalized gold nanoparticles. J. Am. Chem. Soc. 129(37), 11653-11661 (2007).

11 Libutti SK, Paciotti GF, Byrnes AA et al. Phase I and pharmacokinetic studies of CYT-6091, a novel pegylated colloidal gold-rh TNF nanomedicine. Clin. Cancer Res. 16(24), 6139-6149 (2010).

12 Bresee J, Maier KE, Melander C, Feldheim DL. Identification of antibiotics using small molecule variable ligand display on gold nanoparticles. Chem. Comm. 46, 7516-7518 (2010).

13 Bowman M-C, Ballard TE, Ackerson CJ, Feldheim DL, Margolis DM, Melander C. Inhibition of HIV fusion with multivalent gold nanoparticles. J. Am. Chem. Soc. 130 (22), 6896-6897 (2008).
- Reports the use of the same gold nanoparticles of the present study conjugated with a different antiretroviral drug.

14 Simpson CA, Huffman BJ, Gerdon AE, Cliffel DE. Unexpected toxicity of monolayer protected gold clusters eliminated by PEG-thiol place exchange reactions. Chem. Res. Toxicol. 23(10), 1608-1616 (2010).

15 Simpson CA, Salleng KJ, Cliffel DE, Feldheim DL. In vivo toxicity, biodistribution, and clearance of glutathionecoated gold nanoparticles. Nanomedicine 9(2), 257-263 (2013).

16 Chithrani BD, Ghazani AA, Chan WC. Determining the size and shape dependence of gold nanoparticle uptake into mammalian cells. Nano Lett. 6(4), 662-668 (2006).

- Studies the importance of gold nanoparticles' size in their ability to enter cells.

17 Kim JA, Aberg C, Salvati A, Dawson KA. Role of cell cycle on the cellular uptake and dilution of nanoparticles in a cell population. Nat. Nano 7(1), 62-68 (2012).

18 Tkachenko AG, Xie H, Liu Y et al. Cellular trajectories of peptide-modified gold particle complexes: comparison of nuclear localization signals and peptide transduction domains. Bioconjug. Chem. 15(3), 482-490 (2004).

19 Baumann D, Hofmann D, Nullmeier S et al. Complex encounters: nanoparticles in whole blood and their uptake into different types of white blood cells. Nanomedicine 8(5), 699-713 (2013).

20 Solmaz SR, Chauhan R, Blobel G, Mel ák I. Molecular architecture of the transport channel of the nuclear pore complex. Cell 147(3), 590-602 (2011).

21 Feldherr CM, Lanford RE, Akin D. Signal-mediated nuclear transport in simian virus 40 -transformed cells is regulated by large tumor antigen. Proc. Natl Acad. Sci. USA 89(22), 11002-11005 (1992).

22 De La Fuente JM, Berry CC. Tat peptide as an efficient molecule to translocate gold nanoparticles into the cell nucleus. Bioconjug. Chem. 16(5), 1176-1180 (2005).

23 Syed S, Zubair A, Frieri M. Immune response to nanomaterials: Implications for medicine and literature review. Curr. Allergy Asthma Rep. 13(1), 50-57 (2013).

24 Bresee J, Bond CM, Worthington RJ et al. Nanoscale structure-activity relationships, mode of action, and biocompatibility of gold nanoparticle antibiotics. J. Am. Chem. Soc. 136(14), 5295-5300 (2014).

25 Ryan JA, Overton KW, Speight ME et al. Cellular uptake of gold nanoparticles passivated with BSA-SV40 large T antigen conjugates. Anal. Chem. 79(23), 9150-9159 (2007).

26 Tkachenko AG, Xie H, Liu Y et al. Cellular trajectories of peptide-modified gold particle complexes: comparison of nuclear localization signals and peptide transduction domains. Bioconjug. Chem. 15(3), 482-490 (2004).

27 Bender AR, Von Briesen H, Kreuter J, Duncan IB, Rübsamen-Waigmann H. Efficiency of nanoparticles as a carrier system for antiviral agents in human immunodeficiency virus-infected human monocytes/ macrophages in vitro. Antimicrob. Agents Chemother. 40(6), 1467-1471 (1996). 
Shah L, Amiji M. Intracellular delivery of saquinavir in biodegradable polymeric nanoparticles for HIV/aids. Pharm. Res. 23(11), 2638-2645 (2006).

Chattopadhyay N, Zastre J, Wong H-L, Wu X, Bendayan R. Solid lipid nanoparticles enhance the delivery of the HIV protease inhibitor, atazanavir, by a human brain endothelial cell line. Pharm. Res. 25(10), 2262-2271 (2008).

Shiang Y-C, Ou C-M, Chen S-J et al. Highly efficient inhibition of human immunodeficiency virus type 1 reverse transcriptase by aptamers functionalized gold nanoparticles. Nanoscale 5(7), 2756-2764 (2013).

31 Di Gianvincenzo P, Marradi M, Martínez-Ávila OM, Bedoya LM, Alcamí J, Penadés S. Gold nanoparticles capped with sulfate-ended ligands as anti-HIV agents. Bioorg. Med. Chem. Lett. 20 (9), 2718-2721 (2010).

32 Clifford DB, Ances BM. HIV-associated neurocognitive disorder. Lancet Infect. Dis. 13(11), 976-986 (2013).

33 Gray LR, Roche M, Flynn JK, Wesselingh SL, Gorry PR, Churchill MJ. Is the central nervous system a reservoir of HIV-1? Curr. Opin. HIV AIDS 9(6), 552-558 (2014).

34 De Jong WH, Hagens WI, Krystek P, Burger MC, Sips AJ, Geertsma RE. Particle size-dependent organ distribution of gold nanoparticles after intravenous administration. Biomaterials 29(12), 1912-1919 (2008).

35 Sonavane G, Tomoda K, Makino K. Biodistribution of colloidal gold nanoparticles after intravenous administration: effect of particle size. Colloids Surf. B Biointerfaces 66(2), 274-280 (2008).

36 Yim YS, Choi JS, Kim GT et al. A facile approach for the delivery of inorganic nanoparticles into the brain by passing through the blood-brain barrier (bbb). Chem. Commun. (Camb). 48(1), 61-63 (2012).

37 Weiss CK, Kohnle MV, Landfester K et al. The first step into the brain: uptake of NIO-PBCA nanoparticles by endothelial cells in vitro and in vivo, and direct evidence for their bloodbrain barrier permeation. ChemMedChem. 3(9), 1395-1403 (2008).

38 Simpson CA, Agrawal AC, Balinski A, Harkness KM, Cliffel DE. Short-chain PEG mixed monolayer protected gold clusters increase clearance and red blood cell counts. ACS nano 5(5), 3577-3584 (2011).

39 De Boer AG, Gaillard PJ. Drug targeting to the brain. Annu. Rev. Pharmacol. Toxicol. 47, 323-355 (2007).

40 Rapoport SI, Ohno K, Fredericks WR, Pettigrew KD. Regional cerebrovascular permeability to [14c] sucrose after osmotic opening of the blood-brain barrier. Brain Res. 150(3), 653-657 (1978).

41 Etame AB, Diaz RJ, O'reilly MA et al. Enhanced delivery of gold nanoparticles with therapeutic potential into the brain using MRI-guided focused ultrasound. Nanomedicine 8(7), 1133-1142 (2012). 